\title{
Planetary Environments and Origins of Life
}

\section{How to reinvent the study of Origins of Life on the Earth and Life in the Universe under the light of exoplanetology progress}

\author{
Marc Ollivier $^{1, a}$ and Marie-Christine Maurel ${ }^{2, b}$ \\ ${ }^{1}$ Institut d'Astrophysique Spatiale d'Orsay, UMR 8617, CNRS et Université de Paris-Sud \\ ${ }^{2}$ ISYEB, Sorbonne Universités, UMR 7205 et Université Pierre et Marie Curie, Univ Paris 06 F - 75005, \\ Paris, France
}

\begin{abstract}
The present strategies proposed for the search for the origins of life and for extra-terrestrial life are mainly based on Earth-life and its adaptation along geological eras to the Earth environment. These strategies should however face the difficulty that one cannot as yet accurately date the appearance of life on Earth, and consequently one cannot identify a chronology of phenomena related to the transition from the non-living to the living and the dependence of these phenomena on the Earth environment. In this paper, we present new approaches to search for the origins of life on the Earth and next, for life on exoplanets. This study is based on our experience gained at the head of the $\mathrm{CNRS}^{\mathrm{c}}$ interdisciplinary research initiative "Environnements Planétaires et Origines de la Vie $\left(\mathrm{EPOV}^{\mathrm{d}}\right)$, and on the analysis of the projects the initiative funded.
\end{abstract}

\section{Classical paradigm and associated strategies for the detection of life in the universe and their limitations}

Since 1995 and the detection of the first exoplanet around a solar-like star [1], more than a thousand planetary systems have been discovered within our galaxy [2]. Even if $97 \%$ of them have been discovered by indirect techniques of detection (mainly by radial velocity measurements and transit monitoring techniques - for an exhaustive description of the indirect and direct detection methods, see [3]), astronomers have as yet but a partial knowledge of the nature of these worlds, mainly limited to their mass, diameter, orbital parameters and the characteristics of their parent star. For a few of them (about ten giant planets), the presence of an atmosphere has been identified, and partial spectra in the visible, near and mid infrared (several windows from 0.4 to $20 \mu \mathrm{m}$ ) have been obtained by both ground-based and space observatories, leading to the potential identification of $\mathrm{Na}$ [4], $\mathrm{H}_{2}$ [5], $\mathrm{H}_{2} \mathrm{O}, \mathrm{CO}_{2}$ and $\mathrm{CH}_{4}$ [6] in their atmosphere. For all the other planets, and particularly the smaller ones (telluric planets), no observational element so far allows to even confirm the existence of an atmosphere.

\footnotetext{
${ }^{\mathrm{a}}$ e-mail: marc.ollivier@ias.u-psud.fr

be-mail: marie-christine.maurel@upmc.fr

${ }^{\mathrm{c}}$ Centre National de la Recherche Scientifique : French national centre for scientific research

d Planetary environments and Origins of Life
} 
However, the lack of observational characterization of these planets did not prevent scientists from considering the question of their potential habitability, by both theoretical approaches and modelling starting from several assumptions on what life is, and what it may be based on.

The classical assumption is to consider that life on earth is a self-replicating, evolving (with the Darwinian meaning, i.e.: random modifications followed by a selection step) species. This idea leads to the idea notion of "information content" and thus to the need of a "code" to describe the information content. It is assumed that the information is inscribed in carbon chemistry-based processes [7], in liquid water as solvent, because of the very particular characteristics of water (polar, protic, UV-resistant solvent, capable of enabling hydrogen bonds [8]). Consequently, a putative "habitable planet" is usually considered as a planet with liquid water at its surface. Based on these assumptions, the surface temperature and pressure of the planet are thus the most important physico-chemical parameters allowing water to remain liquid. Classically, the surface temperature is estimated thanks to a first order bolometric budget ${ }^{1}$ leading to the determination of the equilibrium temperature of the planet, considered as the surface temperature. The concept of "habitable zone" of a $\operatorname{star}^{2}$ is thus directly extrapolated from these assumptions. A planet can then be considered as being in the habitable zone of its parent star, even if the existence of its atmosphere has still not be confirmed. Semantically speaking, there is thus a huge difference between a habitable planet and a planet within the habitable zone of its parent star.

The second hypothesis usually made to consider the habitability of a planet, is to suppose that life can only be detected if it is developed at a planetary scale and strongly modified the planetary environment, in such a way that the modifications cannot be explained by classical physico-chemical processes (out of equilibrium processes). The development of life at a planetary scale thus requires a considerable amount of raw material, and particularly carbon, in the case a carbon-based life. At a temperate distance from the parent star, carbon exists mainly under its fully oxidized form $\mathrm{CO}_{2}$. Lower oxidation levels are required for carbon-based chemistry ${ }^{3}$ and a powerful process of carbon reduction should be considered under these assumptions. On the Earth, this process is photosynthesis and the result of this process is a large release of oxygen in the atmosphere. That is what happened on the Earth 2.2 billion years ago. Oxygen can thus be considered as a "pollution" of the atmosphere by life. Because of the existence of powerful oxidation processes (with rocks, atmospheric gases...), and the permanent production of fresh reducing material by plate tectonics, the perpetual presence of a huge amount of oxygen in the atmosphere (about $20 \%$ at present), has been considered as a signature of life - a biosignature [9].

Because of the existence of many potential abiotic processes as sources of large quantities of molecular oxygen (e.g.: photolysis of $\mathrm{H}_{2} \mathrm{O}$, of $\mathrm{CO}_{2}$ ), only the presence of a huge quantity of free oxygen in the atmosphere at a given time cannot be considered as a biosignature. Several criteria, some weak and some potentially stronger, have been proposed to identify life remotely. Among them, the simultaneous presence in the atmosphere of $\mathrm{CO}_{2}, \mathrm{H}_{2} \mathrm{O}$, and $\mathrm{O}_{2}$, is certainly one of the most famous, and is known as "the triple signature" [10]. Even if at present no clear process has been identified to generate the triple signature abiotically, this criterion still remains empirical and is

\footnotetext{
${ }^{1}$ A photometric budget is based on the estimation of the incoming and outgoing energy at the planetary scale, over the entire electromagnetic spectral range. Assuming a thermal equilibrium (incoming and outgoing energies are equal), it allows us to determine the average temperature of the planet. Depending on the cloud layer spectral opacities and the potential presence of a greenhouse effect, the equilibrium temperature is either the surface or the atmosphere temperature, or a mixture of both.

${ }^{2}$ The habitable zone of a star is defined by the minimum and maximum orbital distance leading to a planetary surface temperature allowing liquid water to be present at least part of the local year. The habitable zone is often estimated by a bolometric budget assuming or not, the presence of clouds and a greenhouse effect.

${ }^{3}$ Carbon exists at different levels of oxidation from its completely oxidized form $\left(\mathrm{CO}_{2}\right)$ to its fully reduced form $\left(\mathrm{CH}_{4}\right)$. Intermediate oxidation levels allow a large diversity of chemical functions, e.g.: alcohol : R-OH, carboxylic acid R-COOH, amine R-NH2...
} 
limited only to the identification of photosynthesis, as it is known on Earth, i.e: with a large release of free oxygen.

Searching for life thanks only to the triple signature criterion requires that several assumptions are made and has consequences in the way the search is oriented:

- the triple signature cannot be generated abiotically. At present, and after 30 years, no clear mechanism has been identified to generate abiotically the triple signature over a long time (compared to the planetary atmosphere lifetime). However, this lack of identified abiotic mechanism is not a proof that such a mechanism does not exist,

- one must search for something that follows more or less the same evolution mechanism as on the Earth. If the range of suitable environments is very narrow in terms of temperature, pressure, composition of the atmosphere, this supposes that the mechanism should be easy and robust over time and one would expect to detect life with a limited number of planetary candidates harbouring the suited environment. On the contrary, if the range of suitable environments is large, it supposes that the mechanism can adopt several forms. We thus should be sure that the triple signature is still valid,

- with the present definition of the habitable zone based on a bolometric budget of the planetary energy, the ice satellites of Jupiter or Saturn should not be considered as potentially harbouring life because of too cold a surface temperature,

- if life exists under other forms, we will miss them,

- we know neither when life really appeared on the Earth, nor the exact nature of the Earth environment when life appeared. This lack of knowledge of environmental conditions at the beginning of life prevents us from identifying the initial processes that led to first organisms. In addition, it is not possible to identify the complete path life followed from its origins to its present diversity. It is thus very difficult to identify primitive atmospheres that potentially could have led to a photosynthesis-based life.

- at the end, the search for life based on the triple signature can be compared to the "SETI approach" ${ }^{4}$, with the additional uncertainty that it is not possible to completely exclude the possibility of an abiotic origin of $\mathrm{O}_{2}, \mathrm{CO}_{2}$ and $\mathrm{H}_{2} \mathrm{O}$. This means that the potential detection of the triple signature is neither the unquestionable detection of a photosynthetic process nor, of course, the unquestionable detection of life.

The triple signature strategy has however strongly influenced the development of projects towards the remote detection of life on exoplanets. First of all, these projects were oriented towards Earth analogues, around nearby solar-like stars, at temperate distances, and focused on the detection of the triple signature (spectral features of $\mathrm{CO}_{2}, \mathrm{O}_{3}$ and $\mathrm{H}_{2} \mathrm{O}$ respectively at $15 \mu \mathrm{m}, 9.6 \mu \mathrm{m}$ and above $18 \mu \mathrm{m}$ in the thermal infrared and between $0.5 \mu \mathrm{m}$ and $2 \mu \mathrm{m}$ in the visible / near infrared). They excluded the necessary preliminary phase of a purely physico-chemical study of the planetary environment diversity. Furthermore, because it is very difficult to identify pure Earth analogues (earth-size planets at about one astronomical unit from solar-like stars) with present detection techniques, potential projects of spectral characterization have become more complex and expensive as they should include a preliminary phase of detection of suitable targets. Finally, the lack of non-empirical arguments, the cost of the projects, and the speed at which the knowledge of exoplanets field evolves have made this triple signature-oriented strategy difficult to defend and thus quickly obsolete. It is thus necessary to

\footnotetext{
${ }^{4}$ SETI (Search for Extra-Terrestrial Intelligence) is a bunch of projects initiated in the late 60s, aiming at detecting the potential radio-emissions that can spread through the universe from intelligent extra-terrestrial civilizations if they exist. These projects followed several phases of development. At present the search activity strongly decreases because of lack of funding. To date, no detection has been announced.
} 
invent a new approach for the detection and study of life in the universe. This approach is highlighted by the search for the origins of life on Earth, and the characterization of the diversity of planetary environments.

\section{The new horizons of exoplanetology}

Since the discovery of the first extrasolar planets in the mid 90s, the discoveries of the last 15 years have completely shaped a new vision of classical planetology. The diversity of extrasolar systems is illustrated by the detection of more than thousand planets so far [2]. Among the lessons to be learned from these discoveries, one can mention several important points:

- Contrary to our Solar System with well-differentiated telluric and giant planets, with rather different masses and radius, the mass and radius distribution of known extrasolar planets seem to be rather continuous from earth-like (and even smaller) to giant planets [11]. The mass of the core of giant planets appears to be relatively well correlated with the metallicity ${ }^{5}$ of the star [13], tending to confirm the growth of planetesimals scenarios ${ }^{6}$ for the formation process. This process thus leads to a large range of masses and diameters, as a function of the initial protoplanetary disk density and composition.

- whatever the type of star one considers: young, evolved, massive, cool, surrounded by a debris disk, solar-like, and even after it has left the main sequence during the stellar evolution process (stars at the last phase if their life), ... one can find a planet orbiting such a star. This clearly indicates that the planetary formation process, within a protoplanetary disk, is something very common and powerful. The extrapolation based on the known planet distribution within the solar vicinity, tends to show that our galaxy may harbour several billions of planets. Among these planets, the fraction of small planets (telluric planets) seems to be at least several tens of percent, potentially leading to a very huge number of rocky planets [14].

- The diversity of masses and radii for the same type of objects (for instance, giant planets, Neptunelike planets) clearly exhibits a large range of physico-chemical conditions at the planetary surface or in the atmosphere. As a consequence, such diversity tends to lead to a large range of planetary environments.

- Dynamically speaking, the systems are not carved in marble, with planets perpetually evolving on stable orbits. They exhibit permanent internal interactions, leading to a phenomenon referred as orbital migrations [15]. This phenomenon was highlighted by the detection of the first planets that are giant planets on very narrow orbits, with a period of several days only. Because of the very high temperature and too low concentration of dust at this position of the disk, these planets cannot form where they are at present, and should have been brought into the stellar vicinity by this migration process. Several small planets have also been discovered at a distance of only several stellar radii (or are maybe remnants of bigger planets). Assuming the level of stellar wind in the stellar neighbourhood, these planets should have eroded atmospheres, and even may have completely lost their initial atmosphere. This clearly indicates that the climate at a planetary surface may strongly evolve during the entire planetary life and that the notion of "habitability" should also be a function of the planetary history. As a consequence, and because of this migration process, a planet that is formed in the habitable zone of its parent star can be ejected from it. Conversely, a planet at a non-habitable distance from its parent star may nevertheless have been habitable during part of its

\footnotetext{
${ }^{5}$ The metallicity of a star is the mass fraction of elements that are not hydrogen or helium. These elements are supposed to be the raw materials of planetary solid cores

${ }^{6}$ The theory of planetesimals was proposed by Victor Safronov to describe the formation of the Solar System [12]. It supposes the formation of kilometric bodies (the planetesimals) by the coalescence of dust grains from the disk, and then the formation of larger bodies (planetary cores) by gravitational interactions and collision of these bodies.
} 
history.

If one accepts the diversity of planets, it is easy to assume the diversity of planetary environments reflecting both the initial composition of the planetary atmosphere (if it existed or survived) and its evolution during the planetary life. This diversity should now be explored, with specific observatories, to determine the nature and the composition of the atmosphere of a large sample of planets. This work is comparable to the work of naturalists at the beginning of the $18^{\text {th }}$ century, trying to assemble the diversity of life forms on the Earth into a limited number of classes. This work is mandatory before anything else for several reasons:

- to understand the diversity of planetary environments and their evolution,

- to identify the paths planetary evolution can follow, and as a consequence, to see if the Earth is unique (or at least rare) or common,

- to consider the potential environment favourable to the emergence of life and evaluate the potential forms of life it can harbour.

This work should be initiated by the next generation of planetary observatories, such as the James Webb Space Telescope that should be launched in 2018 or EChO (Exoplanet Characterization Observatory) that has been proposed to $\mathrm{ESA}^{7}$ for a potential launch in 2022. These observatories are no longer devoted to the detection of extrasolar systems, but aim at detecting their atmosphere, in order to determine their composition and evolution, at least in the case of the brightest objects.

Knowledge of the diversity of planetary environments will help to build a new strategy for the search of life, based no longer on a more or less argue hypothesis on the universality of photosynthesis, but on the diversity of physico-chemical conditions at the planetary surface.

\section{Towards new strategies for the search of life in the universe}

The diversity of planetary environments, and the potential ability to characterize them in the very near future should lead us to reconsider the question of the search for life and what good biosignatures could be.

This forces us to consider the most generic definition of life, and to identify the minimal assumptions that should be made to avoid entering the field of pure science fiction. Because we consider remote detection of life, thanks to the spectroscopy of the light coming from the planet ${ }^{8}$, the only achievable pieces of information are chemical. It is thus still necessary to consider life and associated processes as something based on chemistry and to try to identify what can be described by pure chemistry, and what requires complex systems capable of taking into account environmental contacts and exchanges. The main difficulty is that a biosignature should be both selective (i.e. it should not lead to the detection of false-positives) and at the same time not be too specific because of a necessary adaptability to the context. A potential method for us would be, once again as the naturalists did at the beginning of the 18th century, to identify and classify all the potential species existing on the Earth, what they produce, what they require, and what potential chemical signature could be used to identify them remotely. We can also take advantage of the most recent research in Systematics Biology, and particularly on questions the notion of Species leads to. Today, more that 200 definitions exist to designate a living species on Earth. What should we then think of the characteristics of what we are searching elsewhere and of the difficulties we encounter in attempting to distinguish what is living from what is not living? This is a huge undertaking, rendered much harder by the fact that the number of photons

\footnotetext{
${ }^{7}$ European Space Agency

${ }^{8}$ Assuming the distance between the Sun and its nearest neighbour, in situ observations thanks to robotic observatories cannot be considered within the next couple of decades.
} 
emanating from planets is very limited and their detection is very difficult because of the presence of the parent star in the same field of view. A biosignature thus can at present only be found in the way life transformed the atmosphere of its harbouring planet, and is a consequence of a given metabolism. The difficulty is once again made harder by the fact that the paths followed by evolution can be multiple. If life should evolve again and follow a path as it did 4 milliard years ago, no one would be able to certify that it would take the same path, leading to the same species, the same cells, the same living beings. This implies that if life exists - or existed elsewhere - benefiting from the same initial planetary conditions, it most likely would not follow the same itinerary, hence it would not have the same history, the same biology. This means certainly that the search for so-called "biosignatures" is in vain. Hence the enigmatic question: how can we possibly recognize and/or identify something that we are unable to conceptualize? This requires a tremendous amount of imagination.

Does it mean that the search for life should not be considered at all? We do not think so. The search for life should first lead to the understanding of what terrestrial life really is, what is the common denominator of all the known metabolisms. This can be done by a multi-disciplinary approach including biology, physics, chemistry, geology, geochemistry, astrophysics ... [16] We know that the building blocks of terrestrial life and chemical processes are common in the Solar System and in the universe (carbon, hydrogen, nitrogen, oxygen, salts,...) This means that the origins of the building blocks of terrestrial life are not a true mystery in the Solar System. However, the next steps, leading to complex structures and associated replication processes (and at the end a complete metabolism) are not well understood. The example of photosynthesis is very illustrative of this problem. It requires several pigments, complex photosystems, numerous enzymes and precise thermodynamical processes creating a major problem for biologists, given the sophistication of photosynthesis in present-day cells. Moreover the expression of molecules of life that is the activity of a biological system at the molecular level depends on the movements of the system rather than on its structure only. In given environmental conditions, the molecule can move from one conformation to another. This suggests an evolutionary selection of specific dynamics as well as of structures with relation to function. Such appropriate dynamics maintain structure and flexibility within the narrow limits required by biological activity. Therefore, to know more about the plausible characteristics of something living elsewhere requires that chemical, biochemical and biophysical parameters be correlated with spectroscopic data. This is a huge program of investigations. Even if we can remotely detect some molecular species in the atmosphere of an exoplanet, it will be very difficult to consider the associated mechanism another way than with models or laboratory work.

Finally, a crucial debate concerns the frontier between what is alive and what is not (for instance are viruses and viroids living species?). Just as what is "pathological" in biology provides us with insight on what is "normal", the space that lies at the border between the living and the non-living will maybe allow us to envisage other forms of life (that we cannot imagine to-day).

The search for life appears thus as something very exciting but also very difficult, and largely hypothetical. Because of the unbelievable consequences of a potential detection of life outside the Earth, particular care should be taken to be sure that what we detect is life and nothing else. This thus requires a step-by-step approach: the next step is and should definitively be the understanding of planetary environments. This step should be completed before further considerations could be envisaged, even if the end may insidiously encourage us to "jump the gun". It is the price that must be paid to keep the search for life in the field of science and not a declaration of faith. "Pleurant, je voyais de l'or, et ne pus boire" ${ }^{9}$, Arthur Raimbaud wrote [17]. Let us not become blind by our most private hopes, but keep working with the force and truth of science.

\footnotetext{
9"Crying, I saw gold and could not drink"
} 


\section{References}

[1] Mayor M. \& Queloz D., Nature, 378, 355 (1995)

[2] The Extrasolar Planets Encyclopaedia, http://exoplanet.eu

[3] Ollivier M., Encrenaz T., Roques F., Selsis F., Casoli F., Planetary Systems - Detection, Formation and Habitability of Extrasolar Planets, Springer, Berlin (2008)

[4] Charbonneau D., Brown T.M., Noyes R.W., Gilliland R.L., ApJ, 568, 377 (2002)

[5] Vidal-Madjar A., Lecavelier des Etangs A., Désert J.-M., Ballester G. E., Ferlet R., Hébrard G., Mayor, M., Nature, 422, 143 (2003)

[6] Swain M. R., Tinetti G., Vasisht G., Deroo P., Griffith C., Bouwman J., Chen Pin, Yung Y., Burrows A., Brown L. R., Matthews J., Rowe J. F., Kuschnig R., Angerhausen D., ApJ, 704, 1616 (2009)

[7] Maurel M-C. and Zaccai G. "Why Biologists should support the exploration of Mars", BioEssays, 23, 977-978 (2001)

[8] Brack A., Origins of Life and Evolution of the Biosphere, 23, Issue 1, pp.3-10 (1993)

[9] Owen T., in "Strategies for the search for life in the universe, M Papagiannis Ed., Reidel, p. 177 (1980)

[10] Léger A., Pirre, M., Marceau T.J., A\&A, 277, p.309 (1993)

[11] Udry S., Santos C.N., An. Rev. of Ast. Astrophys., 45, 397-439 (2007)

[12] Safronov V., Evolution of the protoplanetary cloud and formation of the Earth and planets, NASA-TT-F-677 (1972)

[13] Guillot T., Santos N.C., Pont F., Iro N., Melo C., Ribas I., A\&A, 453, L21-L24 (2006)

[14] Traub W.A., American Astronomical Society, DPS meeting \#44, \#200.02 (2012)

[15] Kely W., Nelson R.P., An. Rev. of Ast. Astrophys., 50, 211-249 (2012)

[16] Maurel M-C., Life elsewhere : a biologist'perspective. Pathways towards habitable planets ASP Conference series, 430, 331-335 (2010)

[17] Raimbaud A., in "Une Saison en Enfer", Poot (Brussels) (1873) 
